Orthopäde $2015 \cdot 44: 742$

DOI 10.1007/s00132-015-3161-5

Online publiziert: 29. August 2015

(c) Springer-Verlag Berlin Heidelberg 2015

\author{
A. Eisenschenk ${ }^{1,2}$ \\ ${ }^{1}$ Abteilung für Hand-, Replantations- und Mikrochirurgie, Unfallkrankenhaus Berlin, Berlin, Deutschland \\ 2 Dieter Buck-Gramcko-Stiftungsprofessur, Hand-und funktionelle Mikrochirurgie, \\ Universitätsmedizin Greifswald, Greifswald, Deutschland
}

\title{
Die Beugesehnenchirurgie
}

In diesem Heft werden der aktuelle Stand und die Möglichkeiten der modernen Beugesehnenchirurgie aufgezeigt. Hierzu haben die Autoren die wesentlichen Aspekte abgehandelt. Es wird die aktuelle Anatomie und Biomechanik der Beugesehnen dargestellt, die Diagnostik der Beugesehnenverletzungen an der Hand erklärt, die immer wieder diskutierten Nahttechniken aufgezeigt, die schwierigen Techniken der Ringbandrekonstruktionen illustriert, die Indikationen und die zu erwartenden Ergebnisse der Beugesehnentransplantationen beschrieben sowie die technisch hoch anspruchsvollen Tenolysen der Beugesehnen bearbeitet, inklusive der großen Bedeutung der Rehabilitation nach Beugesehnenverletzungen und deren Versorgung.

\section{\) Die frühe Übungsbehandlung trägt wesentlich zu besseren Ergebnissen bei}

Nach Langer ,beinhaltet heute die moderne Sehnenchirurgie eine stabile, aber gewebeschonende Sehnennaht, eine Schonung oder Rekonstruktion der Beugesehnenscheide mit verstärkenden Ringbändern und eine möglichst frühzeitige, kontrollierte, passive (und aktive) postoperative Übungsbehandlung zur Steigerung der Sehnenfestigkeit und zur Verringerung der Sehnenadhäsionen“. Die heutigen, deutlich besseren Ergebnisse sind letztendlich auf das neue Konzept einer primären Naht und eine konsequente, frühe postoperative Übungsbehandlung zurückzuführen. Das Ziel einer frei gleitenden Beugesehne nach entsprechender Versorgung ist aber nach wie vor nicht immer erreichbar.

Die Rehabilitation von Beugesehnenverletzungen beginnt unmittelbar nach der operativen Versorgung in Form einer für den Betroffenen vom Operateur selbst festgelegten Nachbehandlung bis zum Erreichen der vollständigen Belastbarkeit. Dieser definierte Nachbehandlungszeitraum von insgesamt 12 Wochen umfasst den Verlauf der physiologischen Sehnenheilung.

Abhängig von Verletzungsmechanismus, Lokalisation der Verletzung, intraoperativer Versorgungsmöglichkeit, Begleitverletzungen sowie die Compliance des Patienten wird anhand der Kenntnis der auf die Sehne einwirkenden Kräfte ein entsprechendes Behandlungsregime individuell festgelegt.

Deshalb ist hier eine Bestandsaufnahme der heutigen Operationsmöglichkeiten das Ziel dieses Hefts unter Berücksichtigung der beiden großen Bereiche, nämlich der operativen Versorgung und der Rehabilitation.

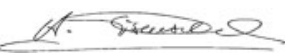

Univ.-Prof. Dr. Andreas Eisenschenk

\section{Korrespondenzadresse}

Univ.-Prof. Dr. A. Eisenschenk

Abteilung für Hand-, Replantations- und Mikrochirurgie

Unfallkrankenhaus Berlin, Berlin andreas.eisenschenk@ukb.de

Interessenkonflikt. A. Eisenschenk gibt an, dass kein Interessenkonflikt besteht. 